\title{
Masked by macrophages
}

Antiretroviral therapy (ART) can suppress HIV-1 replication to undetectable levels in infected individuals; however, interruption of treatment leads to viral rebound and disease progression to AIDS. HIV-1 reservoirs are known to persist in $\mathrm{CD}^{+} \mathrm{T}$ cells, but the role of latently infected tissue macrophages in persistence during ART has been unclear.

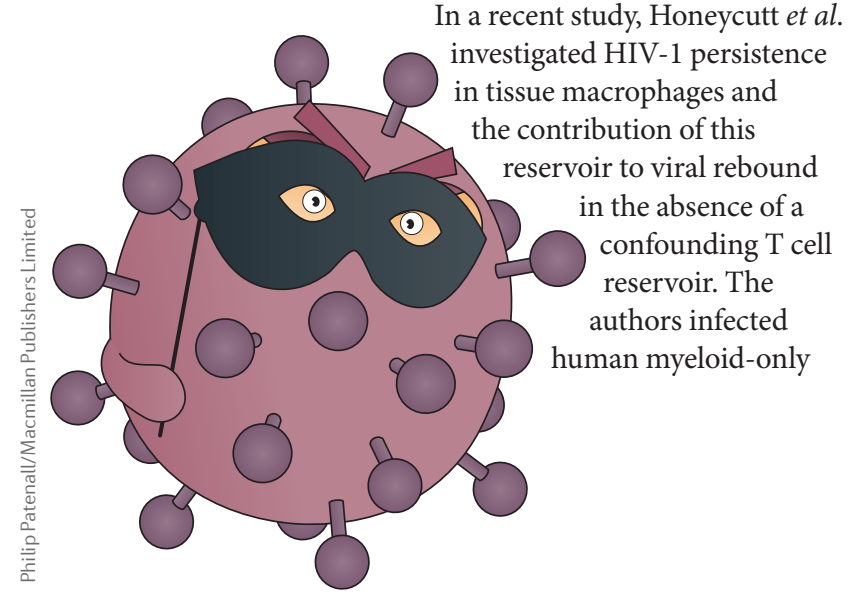

mice (MoM, which are unable to support the development of human $\mathrm{T}$ cells) with macrophage-tropic HIV-1 and they monitored the levels of viral DNA and RNA in macrophages in the absence or presence of ART. Using ultrasensitive quantitative PCR, HIV-1 DNA was found to be integrated into macrophage genomes in the presence of ART, despite undetectable levels of viral RNA. Next, the authors observed that when ART was interrupted in these mice,

\section{Cf}

macrophages may represent a persistent viral reservoir for HIV-1 in vivo approximately one-third of mice had a delayed viral rebound within seven weeks, which is consistent with the establishment of a persistent infection in macrophages. The authors confirmed that human $\mathrm{T}$ cells were absent in these mice and that the rebound virus was replicationcompetent in both macrophages and $\mathrm{T}$ cells.

In summary, this study provides experimental support that macrophages may represent a persistent viral reservoir for HIV-1 in vivo that can re-establish infection after ART interruption.

Ashley York

ORIGINAL ARTICLE Honeycutt, J. B. et al. HIV persistence in tissue macrophages of humanized myeloid-only mice during antiretroviral therapy. Nat.Med. http://dx.doi.org/10.1038/nm.4319 (2017) 